PROCEEDINGS OF THE

AMERICAN MATHEMATICAL SOCIETY

Volume 130, Number 7, Pages 2179-2182

S 0002-9939(02)06307-4

Article electronically published on February 12, 2002

\title{
A CONTINUUM WHOSE HYPERSPACE OF SUBCONTINUA IS NOT $g$-CONTRACTIBLE
}

\author{
ALEJANDRO ILLANES
}

(Communicated by Alan Dow)

\begin{abstract}
A topological space $Y$ is said to be $g$-contractible provided that there exists a continuous onto function $f: Y \rightarrow Y$ such that $f$ is homotopic to a constant function. Answering a question by Sam B. Nadler, Jr., in this paper we construct a metric continuum $Z$ such that its hyperspace of subcontinua $C(Z)$ is not $g$-contractible.
\end{abstract}

\section{Introduction}

A continuum is a compact connected metric space. A map is a continuous function. A topological space $Y$ is said to be $g$-contractible provided that there exists an onto map $f: Y \rightarrow Y$ such that $f$ is homotopic to a constant map. For a continuum $X, C(X)$ (resp., $2^{X}$ ) denotes the hyperspace of subcontinua (resp., nonempty closed subsets) of $X$, with the Hausdorff metric.

Clearly, every contractible space is $g$-contractible. A simple closed curve is an easy example of a $g$-contractible and non-contractible continuum. In fact, the HahnMazurkiewicz Theorem (see [7, Theorem 8.14]) implies that any locally connected continuum is $g$-contractible. The notion of $g$-contractibility was introduced by D. P. Bellamy in [1]. In [5], S. B. Nadler, Jr., studied $g$-contractibility in hyperspaces. He proved that, for any continuum $X, 2^{X}$ is $g$-contractible $([5,3.9]$ or []. Theorem $4.10])$, and if $X$ is a continuum such that $X$ contains an open subset with uncountably many components, then $C(X)$ is $g$-contractible $([5,3.12]$ or [6, Theorem 4.12]). Nadler also asked if $C(X)$ is $g$-contractible for any continuum $X$ ([5 3.10] or [6, Question 4.11]). In this paper we answer Nadler's question in the negative by constructing an example of a continuum $Z$ such that $C(Z)$ is not $g$-contractible.

\section{Auxiliary Results}

Lemma 1. If $C(X)$ is g-contractible, then there exists an onto map $f: C(X) \rightarrow$ $C(X)$ and there exists a map $\psi: C(X) \times[0,1] \rightarrow C(X)$ such that:

- $\psi(A, 0)=f(A)$ and $\psi(A, 1)=X$ for each $A \in C(X)$,

- if $A \in C(X)$ and $0 \leq s \leq t \leq 1$, then $\psi(A, s) \subset \psi(A, t)$.

Proof. Suppose that $C(X)$ is $g$-contractible. Then there exist:

- an onto map $f: C(X) \rightarrow C(X)$,

Received by the editors April 24, 2000 and, in revised form, February 19, 2001.

2000 Mathematics Subject Classification. Primary 54B20.

Key words and phrases. Continuum, $g$-contractible, hyperspace.

(C)2002 American Mathematical Society 
- an element $C \in C(X)$ and

- a map $G: C(X) \times[0,1] \rightarrow C(X)$,

such that $G(A, 0)=f(A)$ and $G(A, 1)=C$ for each $A \in C(X)$.

Since $C(X)$ is arcwise connected ([6, Theorem 1.13]) we can take a map $\alpha$ : $[0,1] \rightarrow C(X)$ such that $\alpha(0)=C$ and $\alpha(1)=X$. Let $F: C(X) \times[0,1] \rightarrow C(X)$ be given by

$$
F(A, t)=\left\{\begin{array}{cl}
G(A, 2 t), & \text { if } t \in\left[0, \frac{1}{2}\right] \\
\alpha(2 t-1), & \text { if } t \in\left[\frac{1}{2}, 1\right] .
\end{array}\right.
$$

Clearly, $F$ is a map such that $F(A, 0)=f(A)$ and $F(A, 1)=X$ for each $A \in$ $C(X)$.

Now, let $\psi: C(X) \times[0,1] \rightarrow C(X)$ be given by

$$
\psi(A, t)=\bigcup\{F(A, s): s \in[0, t]\} .
$$

It is easy to show that $\psi$ has the required properties.

The proof of the following lemma is similar to the proof of Theorem (2) of [2].

Lemma 2. Let $f: X \rightarrow Y$ be an onto map between continua. Let $q \in Y$. If $X$ is connected im kleinen at each point of $f^{-1}(q)$, then $Y$ is connected im kleinen at $q$.

The following result is an easy consequence of Theorem 2 of [3].

Lemma 3. Let $X$ be a continuum and let $p \in X$ be a point such that $X$ is connected im kleinen at $p$. Then $C(X)$ is connected im kleinen at each element $A$ that satisfies $p \in A$.

\section{The EXAMPLE}

The example is constructed in the euclidean plane $\mathbb{R}^{2}$. For each subset $A$ of $\mathbb{R}^{2}$, let $-A$ denote the set $-A=\left\{-p \in \mathbb{R}^{2}: p \in A\right\}$. Let $\theta=(0,0) \in \mathbb{R}^{2}$. Let $X_{0}=\{0\} \times[-1,1]$. For each $n \geq 1$, let $X_{n}=\left\{\frac{1}{n}\right\} \times\left[0, \frac{1}{n}\right]$. For each $n \geq 1$, let $L_{n}$ be a homeomorphic copy of the real line such that: (a) $L_{n} \subset\left(\frac{1}{n+1}, \frac{1}{n}\right) \times[0,1]$, (b) $L_{n} \cap([0,1] \times\{1\}) \neq \emptyset$, and (c) $\operatorname{cl}_{\mathbb{R}^{2}}\left(L_{n}\right)=L_{n} \cup X_{n} \cup X_{n+1}$. Let $Y_{0}=X_{0} \cup\left(\bigcup\left\{X_{n}\right.\right.$ : $n \geq 1\}) \cup\left(\bigcup\left\{L_{n}: n \geq 1\right\}\right)$.

Finally, put $X=\left(-Y_{0}\right) \cup Y_{0}$.

Clearly, $X$ is a continuum (shown on the next page).

Proof. Now we prove that $C(X)$ is not $g$-contractible. Suppose, to the contrary, that $C(X)$ is $g$-contractible. Let $f: C(X) \rightarrow C(X)$ and $\psi: C(X) \times[0,1] \rightarrow C(X)$ be as in Lemma 1. From Lemma 3 , it follows that $C(X)$ is connected im kleinen at any element $A \in C(X)$ such that $A \cap\left[\left(\bigcup\left\{L_{n}: n \geq 1\right\}\right) \cup\left(\bigcup\left\{-L_{n}: n \geq 1\right\}\right)\right] \neq \emptyset$. Thus, if $A$ is an element of $C(X)$ such that $C(X)$ is not connected im kleinen at $A$, then $A$ is contained at some $\operatorname{arc} X_{n}$ or at some $\operatorname{arc}-X_{n}(n \geq 0)$.

Now we prove the following.

If $\mathcal{A}$ is a locally connected subcontinuum of $C(X)$, then $\mathcal{A}$ intersects finitely many sets of the form $C\left(X_{n}\right)$ (and finitely many sets of the form $C\left(-X_{n}\right)$ ).

Suppose, to the contrary, that there is a sequence of integers $n_{1}<n_{2}<\ldots$ such that $\mathcal{A} \cap C\left(X_{n_{k}}\right) \neq \emptyset$ for each $k \geq 1$. Fix an element $B_{k} \in \mathcal{A} \cap C\left(X_{n_{k}}\right)$. Then $B_{k} \rightarrow\{\theta\}$. Thus $\{\theta\} \in \mathcal{A}$. Since $\mathcal{A}$ is locally connected, there exists a subcontinuum $\mathcal{B}$ of $\mathcal{A}$ such that $\{\theta\} \in \mathcal{B}, B_{k} \in \mathcal{B}$ for some $k$ and $H(\{\theta\}, B)<\frac{1}{4}$ for each $B \in \mathcal{B}$, where $H$ is the Hausdorff metric for $C(X)$. Let $B_{0}=\bigcup \mathcal{B}$. From [6] Lemma 1.43], 


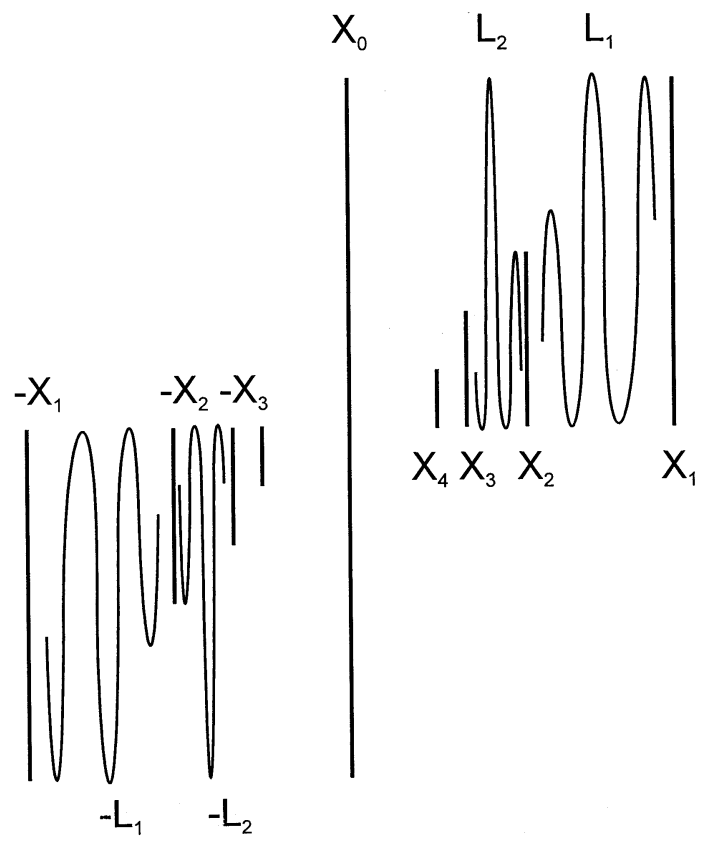

$B_{0}$ is a subcontinuum of $X$ such that $\theta \in B_{0}$, where the diameter of $B_{0}$ is less than $\frac{1}{2}$ and $B_{0}$ intersects $X_{n_{k}}$. From the construction of $X$ one can see that the existence of such $B_{0}$ is impossible. This contradiction completes the proof of the claim.

Next we show that there exists a sequence of subcontinua $\left\{A_{n}\right\}_{n=1}^{\infty}$ of $X$ such that $A_{n} \rightarrow\{\theta\}, f\left(A_{n}\right) \rightarrow\{\theta\}$ and $f\left(A_{n}\right) \subset \bigcup\left\{X_{m}: m \geq 1\right\}$ for each $n$.

Each continuum of one of the forms $X_{i}$ or $-X_{i}$ is an arc, thus the hyperspaces $C\left(X_{i}\right)$ and $C\left(-X_{i}\right)$ are 2-cells ([6, Example 0.54]). Therefore, the sets $f\left[C\left(X_{i}\right)\right]$ and $f\left[C\left(-X_{i}\right)\right]$ are locally connected (see [7, Proposition 8.16]). Given $n \geq 1$, by the previous claim, there exists a positive integer $k_{n}$ such that, $n \leq k_{n}$ and $f\left[C\left(X_{0}\right) \cup C\left(X_{1}\right) \cup C\left(-X_{1}\right) \cup \ldots \cup C\left(X_{n}\right) \cup C\left(-X_{n}\right)\right] \cap C\left(X_{k_{n}}\right)=\emptyset$. Fix a point $p \in X_{k_{n}}$; then it is easy to check that $C(X)$ is not connected im kleinen at $\{p\}$. According to Lemma 2, there exists an element $A_{n} \in C(X)$ such that $C(X)$ is not connected im kleinen at $A_{n}$ and $f\left(A_{n}\right)=p$. Thus, $A_{n}$ is contained in some set of the form $X_{r}$ or in some set of the form $-X_{r}$ for some $r \geq 0$. By the choice of $k_{n}, r \geq n$. This implies that $H\left(\{\theta\}, A_{n}\right)<\frac{1}{n}$. Since $k_{n} \geq n$ and $p \in X_{k_{n}}$, $H\left(\{\theta\}, f\left(A_{n}\right)\right)=H(\{\theta\},\{p\}) \leq \frac{1}{n}$. This completes the construction of $A_{n}$ and proves the claim.

In a similar way, it is possible to construct a sequence of subcontinua $\left\{B_{n}\right\}_{n=1}^{\infty}$ of $X$ such that $B_{n} \rightarrow\{\theta\}, f\left(B_{n}\right) \rightarrow\{\theta\}$ and $f\left(B_{n}\right) \subset \bigcup\left\{-X_{m}: m \geq 1\right\}$ for each $n$.

We are ready to obtain the final contradiction.

Since $f$ is continuous, $f\left(A_{n}\right) \rightarrow f(\{\theta\})$. Thus, $f(\{\theta\})=\{\theta\}$. We know that $\psi(\{\theta\}, 0)=f(\{\theta\})=\{\theta\}$ and $\psi(\{\theta\}, 1)=X$; then the number $t_{0}=\max \{t \in[0,1]$ : $\psi(\{\theta\}, t)=\{\theta\}\}$ is in the interval $[0,1)$. Thus, $\psi\left(\{\theta\}, t_{0}\right)=\{\theta\}$. From the continuity of the map $\psi$, there exists a number $s \in\left(t_{0}, 1\right)$ such that $H(\{\theta\}, \psi(\{\theta\}, s))<\frac{1}{8}$. Hence, $\{\theta\}$ is properly contained in $\psi(\{\theta\}, s)$. 
On the other hand, $\lim \psi\left(B_{n}, s\right)=\psi(\{\theta\}, s)=\lim \psi\left(A_{n}, s\right)$, so there exists $R \geq 1$ such that, for each $r \geq R, \max \left\{H\left(\psi(\{\theta\}, s), \psi\left(A_{r}, s\right)\right), H\left(\psi(\{\theta\}, s), \psi\left(B_{r}, s\right)\right)\right\}<$ $\frac{1}{8}$. Given $r \geq R, \psi\left(A_{r}, s\right)$ is a subcontinuum of $X$ such that it contains the set $\psi\left(A_{r}, 0\right)=f\left(A_{r}\right) \subset \bigcup\left\{X_{m}: m \geq 1\right\}$ and the diameter of $\psi\left(A_{r}, s\right)$ is less than $\frac{1}{2}$. From the construction of $X$, it follows that $\psi\left(A_{r}, s\right)$ is contained in $[0,1] \times[0,1]$. It follows that $\psi(\{\theta\}, s) \subset[0,1] \times[0,1]$. Using $B_{r}$ instead of $A_{r}$, it follows that $\psi(\{\theta\}, s) \subset[-1,0] \times[-1,0]$. Hence, $\psi(\{\theta\}, s)=\{\theta\}$. This is a contradiction that completes the proof that $C(X)$ is not $g$-contractible.

\section{REFERENCES}

[1] D. P. Bellamy, The cone over the Cantor set - continuous maps from both directions, Proc. Topology Conference (Emory University, Atlanta, Ga., 1970), J. W. Rogers, Jr., ed., 8-25. MR 49:6190

[2] R. Engelking and A. Lelek, Cartesian products and continuous images, Colloq. Math. 8, (1961), 27-29. MR 24:A1115

[3] J. T. Goodykoontz, Jr., More on connectedness im kleinen and local connectedness in $C(X)$, Proc. Amer. Math. Soc. 65, (1977), 357-364. MR 56:9475

[4] I. Krzemińska and J. R. Prajs, A non-g-contractible uniformly path connected continuum, Topology Appl. 91, (1999), 151-158. MR 2000a:54044

[5] S. B. Nadler, Jr., Some problems concerning hyperspaces, Topology Conference (V. P. I. and S. U.), Lecture Notes in Math., vol. 375, Springer-Verlag, New York, 1974, R. F. Dickman, Jr. and P. Fletcher, ed., 190-197. MR 51:6692

[6] S. B. Nadler, Jr., Hyperspaces of Sets, A text with research questions, Pure and Applied Mathematics, A Series of Monographs and Textbooks, vol. 49, Marcel Dekker, Inc., New York, 1978. MR 58:18330

[7] S. B. Nadler, Jr., Continuum Theory, An introduction, Pure and Applied Mathematics, A Series of Monographs and Textbooks, vol. 158, Marcel Dekker, Inc., New York, 1992. MR 93m:54002

Instituto de Matemáticas, UnAm, Circuito Exterior, Ciudad Universitaria, México 04510, D.F. MÉXICO

E-mail address: illanes@matem.unam.mx 